\title{
Phase Conjugation of Mid-IR Radiation
}

\author{
V.I. Kovalev \\ Lebedev Physical Institute, Academy of Sciences of the USSR
}

One of the most impressive discoveries in optics in the 1970's was the realization that methods for producing instantaneous light phase conjugation (PC) were feasible. In order to answer the question as to why the PC effect attracts so much attention it is necessary to examine the main features of the PC process.

\section{Fundamental Concepts}

In terms of the complex amplitude of a light wave defined by $E(\vec{r})=|E(\vec{r})|$ $\mathrm{e}^{\mathrm{i} \phi(\vec{r})}, \mathrm{PC}$ corresponds to a change in sign of the phase of the signal wave $E_{s}(\vec{r})$. In mathematical terms, this is the transition from $E_{s}(\vec{r})$ to the complexconjugate wave $E_{s}^{*}(\vec{r})$ in such a way that the conjugated wave complex amplitude $E_{c}(\vec{r})=E_{s}^{*}(\vec{r})$ or $\phi_{c}(\vec{r})=-\phi_{s}(\vec{r})$. An arrangement that allows one to realize the conjugate wave is called a phaseconjugate mirror (PCM).

One of the most interesting properties of a conjugated wave is its ability to reproduce the wavefront structure of an initial wave, distorted on passing through a medium, simply by making a backward pass through the same medium (Fig. 1). The majority of its possible applications are based on this property including beam divergence improvement up to the diffraction limit for high-power lasers. In essence, it is beam divergence that limits the distance over which effective transmission can be made of laser energy, while the interaction efficiency of a laser beam with matter is determined by the laser energy concentration and its distribution in the irradiated zone. Both are directly related to beam divergence.

The main obstacle to achieving minimum (or diffraction-limited) beam divergence is the existence of non-uniformities and aberrations in the optical elements and active media of lasers and laser systems. This problem can be overcome in principle using high-quality optics, careful selection of the laser cavity modes, and space filtration of the

Dr. V.I. Kovalev is a senior researcher with the P.N. Lebev Physical Institute, Academy of Sciences of the USSR, Leninsky Prospect 53, 117924 Moscow, USSR.

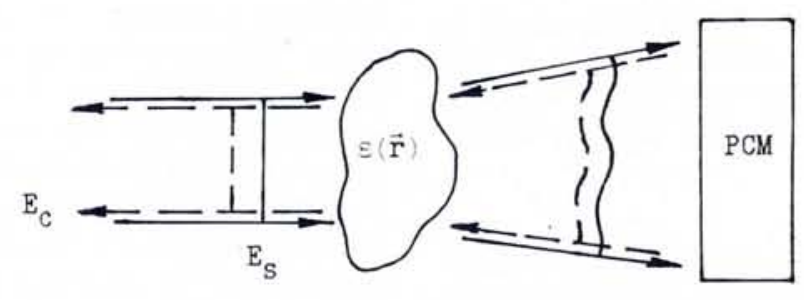

Fig. 1 - Schematic illustration of the compensation of spatially-dependent phase distortions by a PCM. An incident plane wave $E_{s}$ passes through a medium with a non-uniform $\varepsilon(\vec{r})$. The wave with a distorted wavefront is reflected by a PCM to give a wave which, after passing again through the original distorting medium, emerges with the same wavefront, $E_{c}$ as the incident wave. The arrows indicate the local plane wave components of each wavefront.

output radiation. However, these approaches lead to decreases in the laser output power and efficiency and to a generally unacceptable increase in cost. By using PC it becomes possible to obtain diffraction-limited beam divergence essentially without any decrease in the laser efficiency, even with lowquality optical elements. The distribution of intensity over the beam crosssection can also be formed and stabilized so as to produce self-pointing and self-tracking of radiation to a target in laser fusion installations, in laser lithography, mapping, cutting, and other laser technologies.

A solution to the divergence problem is particularly important for lasers in the mid-IR range using gases such as $\mathrm{Xe}$, $\mathrm{HF}, \mathrm{DF}, \mathrm{CO}$ and $\mathrm{CO}_{2}$. These lasers are at present the most powerful and effective available and they are widely used in both scientific laboratories and industry. Because of the relatively long wavelength $\lambda$, the diffraction-limited divergence of the beam is significant and even a small deterioration is undesirable.

The main requirements for the $\mathrm{PC}$ system are: 1) it provides high quality phase conjugation; 2) the response is sufficiently rapid to compensate for optical inhomogeneities that vary with time; 3 ) the conversion efficiency of the signal wave to the phase-conjugated wave is sufficiently high. Several methods for producing phase conjugation have been suggested and developed but the most widespread are stimulated Brillouin scattering (SBS) and four-wave mixing (FWM). The chief merit of the former is its exceptional simplicity: it is only necessary to have an active medium and an optical system to guide the laser radiation into it. Socalled self-conjugation takes place, the signal wave creating in the medium a structure of acoustic vibrations that gives rise to the phase conjugated component in the backscattered radiation. This process requires, however, a highintensity signal wave; quality is determined here by the interaction geometry and it can approach $90 \%$. The response time is determined by the decay of acoustic phonons, which as a rule is not less than $1 \mathrm{~ns}$. The maximum reflectivity $R_{\mathrm{m}}$ that can be achieved depends on the energies of the photons $(\hbar \omega)$ and the phonons ( $\hbar \Omega$ ) and can be expressed as $R_{\mathrm{m}} \cong 1-\Omega / \omega$. Since $\Omega \ll \omega$, $R_{\mathrm{m}}$ is close to unity.

In contrast to the visible and near IR wavelength ranges where the main effort has been directed towards the SBS method, in the mid-IR range it is four-wave mixing that has attracted most attention. With FWM, two counter propagating pump waves must be directed into a non-linear medium simultaneously with a signal wave (Fig. 2), but the advantage is that effective conversion of the signal wave to a conjugated wave is possible even for very weak signals. Indeed, reflectivities greater than unity may be realized. The response time is defined by the non-linearity mechanism of the medium and can vary from $10^{-15} s$ up to several seconds. To obtain high quality PC it is necessary 


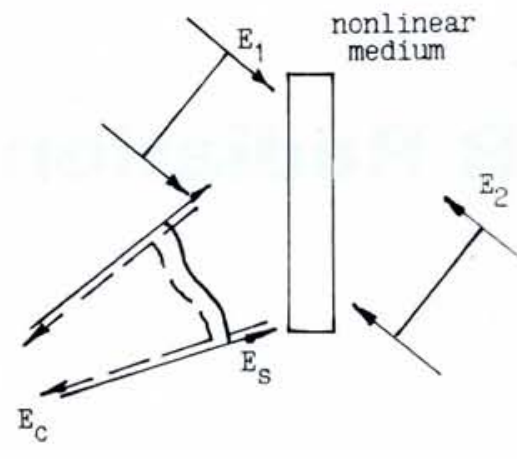

Fig. 2 - The geometrical arrangement for reverse optical phase conjugation by fourwave mixing. The pump waves $E_{1}$ and $E_{2}$ are counter-propagating and have conjugated wavefronts.

to produce and maintain powerful counterpropagating pump waves with exactly conjugate wavefronts.

\section{Phase Conjugation by SBS}

The main parameters which determine the possibility of effective SBS excitation are the steady-state gain increment $G=g l_{0} \ell$ where $g$ is the steadystate gain, $I_{o}$ is the intensity of the exciting radiation, $\ell$ is the interaction length, and $\tau$ the decay time of hypersonic waves in the medium. The scattered intensity $I_{s}$ is related to $G$ by the expression $I_{s}=I_{s}^{s p} \exp \mathrm{G}$, where $I_{s}^{s p}$ is the spontaneously produced density fluctuation in the non-linear medium. It is necessary to have $G \geq 25$ for a reflectivity approaching unity $\left(I_{s} \cong I_{o}\right)$ in the visible range because in a medium in thermodynamic equilibrium $I_{s}^{s p} \cong$ $\left(10^{-10}-10^{-12}\right) I_{0}$. However, in the mid-IR, as $I_{s}^{s p} \sim \lambda^{-4}$ (Rayleigh's law), $G$ has to be even larger if not by very much (approximately 1.5 times when $\lambda$ increases from $\cong 0.7 \mu \mathrm{m}$ to $\cong 10 \mu \mathrm{m}$ ). Much more important is the quadratic dependence of $\tau$ on $\lambda$. For backward SBS, $\tau \geq 1 \mathrm{~ns}$ for liquids and solids and $\geq 10 \mathrm{~ns}$ for gases in the visible range $(\lambda \leq 1 \mu \mathrm{m})$, while in the mid-IR range $(\lambda \geq 10 \mu \mathrm{m}) \tau$ increases up to more than 0.1-1 $\mu$ s. The larger $\tau$ imposes more severe demands on the coherence of the exciting SBS radiation which are difficult to realize in lasers in combination with a high enough output power. Moreover, despite the fact that the steady-state SBS gain and the threshold exciting intensity are independent of $\lambda$, the threshold energy density increases in proportion to $\tau$. This energy density normally exceeds the damage thresholds of available IR transparent SBS active media, as was also the situation for the visible range in the midsixties when investigations on SBS started. Progress in producing damage resistant optical materials has overcome the difficulty and it can be hoped that the same progress will be made in mid-IR transmitting optical materials.

\section{Phase Conjugation by FWM}

The main feature of phase conjugation by four-wave mixing is the quadratic dependence of the power reflectivity $R$ on $\lambda$ :

$$
R \cong[\delta \varepsilon l / \lambda]^{2}
$$

where $\delta \varepsilon$ is the non-linear change of the dielectric functions $\varepsilon$ in the medium. Under the same steady-state conditions, this implies that $R$ in the mid-IR range is more than 100 times less than in the visible range. Such a drop cannot be compensated for by increasing the length $\ell$ because of linear absorption which may be significant in mid-IR transmitting media. Achieving large $R$ therefore demands media with large $\delta \varepsilon$.

Any medium with a complex dielectric function $\varepsilon$ dependent on the radiation characteristics is a candidate so FWM reflection and the $\mathrm{PC}$ effect in the mid- $\mathrm{IR}$ are being investigated in gases, liquids and solids by exploiting: 1) the electronic, orientational, strictional, and thermal non-linearities; 2) the vibrational-rotational transitions of molecules and the impurity levels of solids; 3 ) the dependence of the surface reflectivity and profile on the radiation parameters, etc. The variety of non-linearity mechanisms and non-linear media provides a wide range of possibilities for FWM reflection according to intensity, pulse length, spectral composition and state of polarization.

In all circumstances it is necessary to use a medium with a short decay time of the non-linear response, $\tau$. The shortest $\tau$ is associated with the anharmonic motion of the bound electrons because this non-linearity is not associated with a population change of any energy level in the medium. The absolute value of $\tau \cong 10^{-14}-10^{-15} \mathrm{~s}$ corresponds to the atomic time $a / v$ where $a$ is the atomic size, $v$ is the electron velocity in the atom. Such a short $\tau$ allows us to obtain effective FWM reflection of a signal which is not coherent with the pump waves and even of non-monochromatic waves: this is very important for practical applications.

The non-linear change of $\varepsilon$ due to the anharmonic motion of bound electrons is proportional to the electric field $E$ of the light wave $\delta \varepsilon=\chi_{b}{ }^{(3)}|E|^{2}$, where $\chi_{\mathrm{b}}{ }^{(3)}$ is the third-order non-linear optical susceptibility (see Mace D.A.H. and Adams M.J., Aspects of Non-linear Optics, Europhys. News 18 (1987) 48). The values of $\chi_{b}{ }^{(3)}$ are practically independent of the light field frequency $\omega$ for photon energies less than the band gap energy $E_{\mathrm{g}}$ of the medium, and they reach the highest values in semiconductors with small $E_{\mathrm{g}}$ (see Table). As a rule, these materials are transparent in the mid-IR and, based on non-linearity of $\varepsilon$, the PC effect by FWM in the mid-IR has indeed been observed in $\mathrm{Ge}[1,2]$.

In semiconductors with a moderate free-carrier concentration, non-linearities due to the non-linear motion of mobile carriers take place. This arises from the non-parabolic dependence of the conduction band energy $W$ on the momentum $p$. The third-order susceptibility $\chi_{n}{ }^{(3)}$ in this case is given by the $p^{4}$ term in the power series expansion of $W(p)$. Since the momentum of an electron in any energy band in the electric field of a light wave is proportional to $\omega^{-1}, \chi^{(3)}$ proves to be strongly dependent on $\omega: \chi_{n}{ }^{(3)} \sim \omega^{-4}$. Calculations on the basis of the "Kane model" for narrow-gap semiconductor band structures give the $\chi_{n}{ }^{(3)}$ dependence on the semiconductor parameters as

$$
\chi_{n}{ }^{(3)} \sim N / E_{g} m^{* 2},
$$

where $N$ is the free-carrier density, $m^{*}$ is the effective mass of the electron at the bottom of the conduction band. The expression shows that at fixed $N, \chi_{n}{ }^{(3)}$, like $\chi_{b}{ }^{(3)}$, reaches a maximum in semiconductors with small $E_{\mathrm{g}}$. Some typical susceptibility values for $N \cong 2 \times 10^{16}$ $\mathrm{cm}^{-3}$ and a light frequency $\omega$ corresponding to $\lambda=10.6 \mu \mathrm{m}$ are presented in the Table 1. Except for $\mathrm{Ge}$, where the

Table - Third-order Non-linear Susceptibilities in esu

\begin{tabular}{|c|c|c|c|c|c|}
\hline Material & $E_{g}, \mathrm{eV}$ & $\chi_{\mathrm{b}}^{(3)}$ & $\chi_{\mathrm{n}}^{(3)}$ & $\chi_{\mathrm{p}}^{(3)}$ & $\chi_{\mathrm{s}}^{(3)}$ \\
\hline $\mathrm{Ge}$ & 0.67 & $10^{-10}$ & $\cong 10^{-11}$ & $\cong 10^{-9}(6)$ & - \\
\hline $\mathrm{InAs}$ & 0.35 & $\cong 4 \times 10^{-10}$ & $2.5 \times 10^{-7}$ & $10^{-7}(3)$ & - \\
\hline $\mathrm{InSb}$ & 0.17 & $\cong 5 \times 10^{-9}$ & $4 \times 10^{-7}$ & $\cong 10^{-5}(2)$ & $\begin{array}{c}9 \times 10^{-2} \\
(5 \mu \mathrm{m})\end{array}$ \\
\hline $\mathrm{HgCdTe}$ & 0.138 & - & $\cong 4 \times 10^{-7}$ & $5 \times 10^{-6}(2)$ & $\begin{array}{c}3 \times 10^{-2} \\
(10 \mu \mathrm{m})\end{array}$ \\
\hline
\end{tabular}


nonparabolic dependence of the conduction band is small, $\chi_{\mathrm{n}}{ }^{(3)} \gg \chi_{\mathrm{b}}{ }^{(3)}$, and this has been confirmed experimentally $[3,4]$.

This non-linearity, like the non-linearity of bound electrons, is not accompanied by a change in the population of any energy level. So the decay time $\tau$ is short enough. For $N \cong 10^{16}-10^{17} \mathrm{~cm}^{-3}$, $\tau$ is governed by the scattering of electrons by phonons and is, typically $\cong$ $10^{-11}-10^{-12} \mathrm{~s}$, being much larger than the period of a light wave in the mid-IR. The non-linearity therefore does not allow one to perform phase conjugation of a signal with an arbitrary state of polarization so there is a limit to the FWM reflection efficiency for incoherent and non-monochromatic light waves.

It is well known that a large enhancement of the non-linear change of $\varepsilon$ takes place when a redistribution of energy level populations in a medium is involved. In the mid-IR, various types of transition are possible including valenceto-conduction band and intra-band transitions, transitions between the electronic levels of impurities, or between impurity levels and bands or excited levels in semiconductors and dielectrics, vibrational-rotational transitions in molecules, etc. The dominating mechanism may involve: 1) a change of plasma frequency $\omega_{\mathrm{p}}$ by photogenerated free electrons $\left.\left(\delta \varepsilon \cong \omega_{\mathrm{p}}^{2} / \omega^{2}\right) ; 2\right)$ saturation of the corresponding transitions. In both cases not only the real but also the imaginary part of $\varepsilon$ changes, while for the second case it is known that the imaginary part plays the main role in the FWM process.

In the first case, the dependence of $\delta \varepsilon$ on $\omega$ can be expressed as:

$$
\delta \varepsilon \sim\left(I / m_{\text {eh }}^{*} \omega^{2}\right)\left(\eta \beta \tau I^{n-1} / \hbar \omega\right)
$$

Here $m_{\mathrm{eh}}^{*}$ is the reduced effective mass of the electron-hole pair at the band edge, $\eta$ is the quantum efficiency of electron-hole pair generation per absorbed photon, $\beta$ is the coefficient of $\mathrm{n}$-photon absorption, $\tau$ is the recombination time of the electron-hole plasma. The second term is the photo-induced density of free carriers due to n-photon absorption. The equation implies that: 1) $\delta \varepsilon_{\mathrm{p}} \sim \omega^{-3}$, i.e. $\delta \varepsilon_{\mathrm{p}}$ increases rapidly with $\lambda$;

2) $\delta \varepsilon_{\mathrm{p}} \sim \tau$. Since $\tau$ in semiconductors can be changed over a wide range (e.g. in InSb from $\leq 10^{-9}$ to $\cong 10^{-5} \mathrm{~s}$ ) depending on the material quality, the dopant type and concentration, and the sample temperature, the value of $\delta \varepsilon_{\mathrm{p}}$ can be changed over the same range; 3) $\chi_{\mathrm{p}}{ }^{(3)}$ is dependent on $E$ (or $I=|E|^{2}$ ) in contrast to the non-resonant nonlinearities. Depending on the number of photons, $n$, needed to photogenerate electron-hole pairs, in the power series expansion of $\delta \varepsilon$ on $E$, the major contribution to $\delta \varepsilon_{\mathrm{p}}$ is the term which is proportional to $\chi^{(2 n+1)} E^{2 n}$. The Table gives the values of $\chi_{p}{ }^{(3)} \cong\left[\chi^{(2 n+1)} E^{2(n-1)}\right]$ corresponding to the highest achievable $E$ (or I) for the materials listed (the corresponding $n$ value is in brackets).

Much higher values of $\chi_{p}{ }^{(3)}$ are typical of the non-linearities brought about by saturation effects $\left(\chi_{\mathrm{s}}^{(3)}\right)$ of resonant one-photon transitions. For example, $\chi_{\mathrm{p}}{ }^{(3)}$ attains the "giant" value of $\cong 10^{-1}$ esu in semiconductors where the strong effect is caused by a change in the semiconductor gap by the BursteinMoss shift. This takes place at high concentrations of free carriers when the Fermi energy level is moved into the conduction band and the transitions to states below the Fermi energy level are blocked by the Pauli principle.

Just as with interband transitions in semiconductors, many one-photon resonant transitions in semiconductors and molecular gases are used in experiments for PC by FWM in the mid-IR [4, 5]. All effects are connected with the transitions between resonant energy levels and take place in the vicinity of the corresponding resonant frequency without manifesting any specific dependence on $\omega$. All the observed pecularities arising in the mid-IR are brought about by specific features of the materials.

The decay time $\tau$, of non-linearities involving the redistribution of populations is limited by the rates of energy build-up and relaxation. The range of decay times is extremely wide: from about 10 ps (for $\mathrm{SF}_{6}$ introduced into a $\mathrm{NaCl}$ or $\mathrm{KCl}$ matrix [6]) up to about a second (equivalent to the recombination time in Si).

\section{Concluding Remarks}

This short review on non-linear media for FWM in the mid-IR is not exhaustive, but it is important to note one property that is common to all. Despite the vast differences in the mechanisms and in the magnitudes of the individual nonlinearities, the value of $\chi^{(3)} / \tau$ is always between $10^{4}$ and $10^{5} \mathrm{esu} / \mathrm{s}$. Media with high $\chi^{(3)}$ have large $\tau$, and the increase in $\tau$ not only leads to a limitation on the change in rate constants of optical inhomogeneities (which can be compensated for by a PCM), but also places more severe demands on the monochromaticity and mutual coherence of the signal and pump waves.

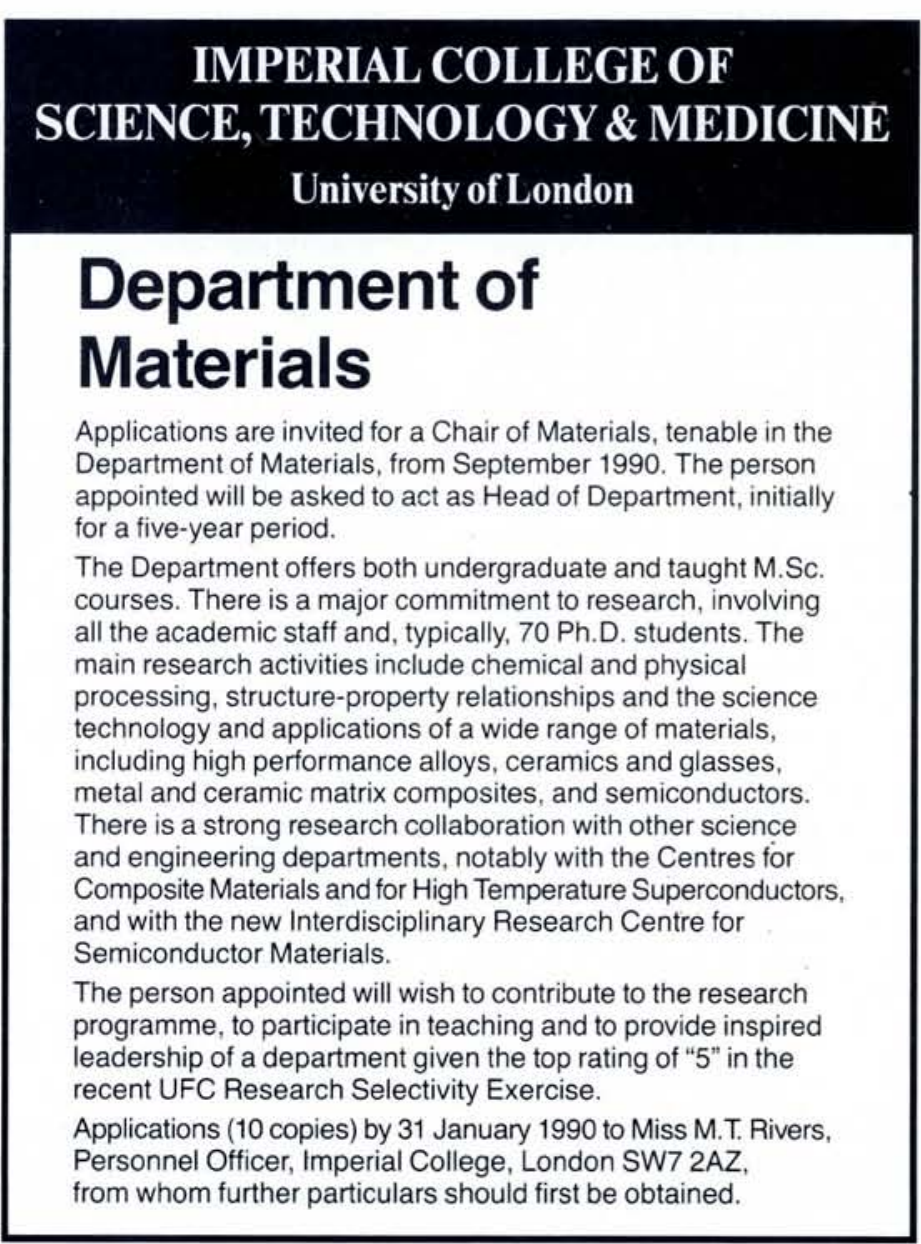


Fig. 3 - Phase conjugate reflectivity as a function of the pump wave intensity for InSb (1), InAs (2) and $\mathrm{Ge}$ (3) samples of 0.05, 0.11 and $1.0 \mathrm{~cm}$ thickness respectively. The vertical dashed line indicates the damage threshold of the samples for a $\mathrm{CO}_{2}$-laser pulse length of $\cong$ $0.1 \mu s$ [4].

The magnitude of $\delta \varepsilon$ is governed by the type of non-linearity and the characteristics of the non-linear medium as well as by the effective light wave intensity. In dielectrics and wide-gap semiconductors, this intensity is limited, as a rule, by optical damage to the material. In the narrow-gap semiconductors, the limiting phenomenon is non-linear absorption owing to the $n$-photon induced generation of electron-hole pairs. The cross-section for this is defined by the cross-section of the resonant transition between sub-bands of light and heavy holes in the valence band. That is why it is so large $\left(\cong 10^{-15} \mathrm{~cm}^{2}\right)$. As a result, the growth of $R$ with an increasing pump wave intensity in narrow-gap semiconductors reaches a maximum $R_{\mathrm{m}}$ determined by the non-linear absorption (see Fig. 3). If this is taken into account, $R_{\mathrm{m}}$ in the narrow-gap semiconductors becomes a characteristic property defined by the sample's nonlinear susceptibility, the constant of non-linear absorption, and the coefficient of the linear absorption [4]. Calculations for the materials listed in the Table show that only for the semiconductors $\ln A s$ and $\operatorname{lnSb}$ is $R_{\mathrm{m}} \geq 1$ at wavelength of $10.6 \mu \mathrm{m}$.

In addition to limiting $R$ by FWM,

\section{REFERENCES}

[1] Bergman E.E., Feldman B.J., Bigio J.P. and Fisher R.A., Opt. Lett. 3 (1978) 82.

[2] Depatie D. and Haueisen D., Opt. Lett. 5 (1980) 252.

[3] Khan M.A., Kruse P.W. and Ready J.P., Opt. Lett. 5 (1980) 261.

[4] Basov N.G., Kovalev V.I., Musaev M.A. and Faizullov F.S., Phase Conjugation of Laser Emission, Proc. Lebedev Phys. Institute 172 (Nova Science Publishers: 1988) 147.

[5] Jain R.K., Opt. Engineering 21 (1982) 199.

[6] Greene L.H., Sievers A.J. and Figueira J.F., IEEE J. Quantum Electr., QE-17 (1981) 446.

non-linear absorption also has another important role to play. One of the fundamental causes for the deterioration of $\mathrm{PC}$ quality is distortion of the pump wavefronts by the self-focussing effect (Fig. 4). Non-linear absorption smoothes the transverse distribution of intensity for the pump waves, allowing a reduction in the self-focussing wavefront distortions. Numerical calculations demonstrate that FWM of a beam with a Gaussian transverse beam profile in a cubic non-linear medium having a non-linear absorption provides a PC quality of about $95 \%$ for $R \geq 1$. (In a non-absorbing medium, the PC quality is $\cong 30 \%$ ceteris paribus.)

Other important characteristics of the reflectivity are the range of the signal wave intensities (the dynamic range), the potential for polarization reconstruction, the spectral and spatial selectivity, etc. [4]. Most of these, however, are practically independent of $\omega$, so they do not show any unique features in the mid-IR.

It can be concluded that phase conjugation in the mid-IR is sufficiently well understood that one can start to apply it in practical lasers and laser systems. This will be the main thrust of future activity in the field.

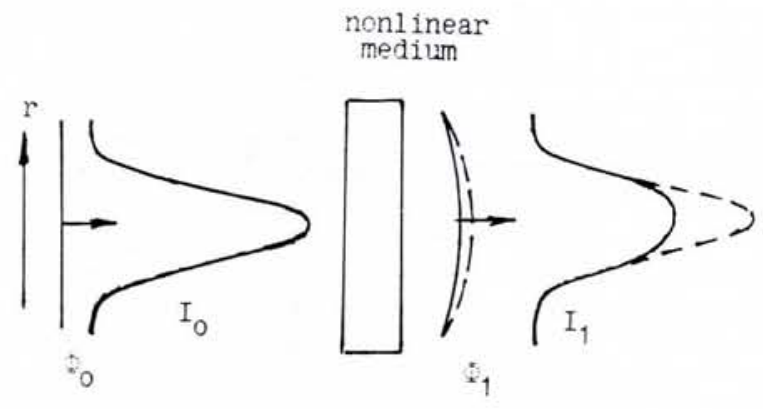

Fig. 4 -Schematic representation of the reduction of self-focussing wavefront distortion in a non-linear absorbing medium for a beam with non-uniform intensity profile. The dashed lines on the right represent the profiles of the intensity land the phase $\phi$ for a non-absorbing medium.

14 Europhys. News 21 (1990)

\section{Beryllium Brings its Benefits}

Before being shut down towards the end of 1989 for general maintenance and the replacement of a suspect toroidal field coil, JET produced discharges of record breaking quality, primarily as a result of the reduction in high $Z$ impurities brought about by the installation of beryllium tiling and a flush of beryllium over the surface of the vacuum vessel. The results have triumphantly justified the conviction of the JET Director, PaulHenri Rebut who was advocating as early as 1985 the provision of Be surfaces to help reduce contamination of the plasma. At the time, however, it was judged prudent to start with carbon tiles as they represented no health hazard. These certainly gave big improvements in performance over the high nickel surfaces, but contamination of the plasma with carbon was still a restricting factor.

The best individual figures that have been obtained now are a temperature, $T$, of 280 MK, a plasma density, $n$, of $4 \times 10^{20} / \mathrm{m}^{3}$ and an energy confinement time, $\tau$, of $1.8 \mathrm{~s}$. The combined figure of merit, the product $T n \tau$, for individual discharges has been pushed up to $6 \times 10^{21} \mathrm{MK} \mathrm{m}^{-3} \mathrm{~s}$, an improvement of a factor 2.4 compared with the best previous performances. This is within a factor 10 of the figure associated with ignition.

Originally set up as a 12-year project starting in June 1978, agreement for a three-year extension to JET has already been approved, but this will clearly not be sufficient to exploit fully the potential of the device. The Director is currently advocating the continuation of experiments on impurity control through 1992-1994 followed by tritium operation up to the end of 1986 , by which time radiation damage is likely to have taken its toll of the main coils.

The present replacement exercise, scheduled to last nine months, will be instructive in giving experience on making a major breach in the vacuum chamber whilst working with partially restricted access — light radioactive contamination and exposed $\mathrm{Be}$ surfaces - and should allow a better appreciation to be made of the full range of problems that tritium operation will bring. 